\title{
G34.24+0.13MM: A DEEPLY EMBEDDED PROTO-B STAR
}

\author{
T. R. HUNTER \\ Smithsonian Astrophysical Observatory, MS-78, 60 Garden Street, Cambridge, MA 02138 \\ AND \\ G. Neugebauer, D. J. Benford, K. Matthews, D. C. Lis, E. Serabyn, and T. G. Phillips \\ California Institute of Technology, MS 320-47, Pasadena, CA 91125 \\ Received 1997 October 21; accepted 1997 November 26; published 1998 January 14
}

\begin{abstract}
By means of millimeter and submillimeter imaging, we have identified a massive protostellar object that coincides with a methanol maser and is not detectable in the continuum at centimeter wavelengths. Located 84" $(1.5 \mathrm{pc})$ southeast of the ultracompact H II (UCHII) region G34.26+0.15, the new object G34.24+0.13MM was discovered in a wide-field $350 \mu \mathrm{m}$ continuum image obtained with the Submillimeter High Angular Resolution Camera (SHARC) at the Caltech Submillimeter Observatory (CSO). Interferometric imaging at 225.7 and 110.7 $\mathrm{GHz}$ continuum has determined more precisely the position and angular diameter (2".0, or $7600 \mathrm{AU})$ of the object. No source was detected at that position in 1.2-3.7 $\mu \mathrm{m}$ imaging or 10 and $20 \mu \mathrm{m}$ photometry. Our observations are consistent with a cool dust core with temperature $\sim 50 \mathrm{~K}$, total gas mass $100 M_{\odot}$, and total luminosity in the range of 1600-6300 $L_{\odot}$. Considering the high luminosity and lack of compact radio continuum emission, we conclude that this core probably contains a deeply embedded proto-B star.
\end{abstract}

Subject headings: stars: formation — infrared: stars — ISM: individual (G34.24+0.13)

\section{INTRODUCTION}

As a consequence of their presumed youth, ultracompact $\mathrm{H}$ II (UCHII) regions provide a good tracer of current massive star formation (Wood \& Churchwell 1989). Recent high-resolution millimeter-wave images of UCHII regions have revealed the high-mass equivalent of "class 0" protostars. Examples include the Turner-Welch object near $\mathrm{W} 3 \mathrm{OH}$ (Wilner, Welch, \& Forster 1995; Turner \& Welch 1984) and the compact methyl cyanide core near the G31.41+0.31 UCHII region (Cesaroni et al. 1994). Because UCHII regions typically lie at distances of several kiloparsecs, the identification of accompanying high-mass protostars requires both good sensitivity and high spatial resolution. In addition, an optically thin tracer is required to probe through the large extinction toward the giant molecular cloud cores that harbor young high-mass stars. Submillimeter continuum emission from cool dust is a good tracer of protostars because it remains optically thin at high column densities $\left(N_{\mathrm{H}} \lesssim 10^{25} \mathrm{~cm}^{-2}\right)$ (Mezger 1994). However, until recently, constructing images with single channel detectors has been time consuming. With the advent of bolometer array cameras at large telescopes, such as SHARC at the CSO (Wang et al. 1996), submillimeter continuum images can now be obtained quickly.

During the commissioning of SHARC, an imaging survey of 17 fields containing UCHII regions was completed (Hunter 1997). One of the fields observed was the H II complex with Galactic coordinates G34.3+0.2. If the distance to G34.3+0.2 is $3.7 \mathrm{kpc}$ (Kuchar \& Bania 1994), the bolometric luminosity of the entire region is $5.9 \times 10^{5} L_{\odot}$ based on the IRAS flux densities (Wood \& Churchwell 1989). At the core of G34.3+0.2 sits the prototypical cometary UCHII region G34.26+0.15 (Reid \& Ho 1985) with an adjacent cluster of $\mathrm{H}_{2} \mathrm{O}$ masers (Fey et al. 1994; Benson \& Johnston 1984) and OH masers (Gaume \& Mutel 1987; Garay, Reid, \& Moran 1985). Interferometric observations of $\mathrm{CH}_{3} \mathrm{CN}$ (Akeson \& Carlstrom 1996) and $\mathrm{NH}_{3}$ (Heaton, Little, \& Bishop 1989) indicate a concentration of dense gas on the east side of the UCHII region. In addition to these centers of activity, we have identified a separate core 84" away (G34.24+0.13) exhibiting compact continuum and molecular line emission. This core is unresolved from the UCHII region in all four bands of HIRESprocessed IRAS images (Hunter 1997). A subsequent literature search revealed the presence of a class $\mathrm{II} \mathrm{CH}_{3} \mathrm{OH}$ maser at this position at $6.6 \mathrm{GHz}$ (Caswell et al. 1995b) and $12.2 \mathrm{GHz}(\mathrm{Ca}-$ swell et al. 1995a). In this Letter, we combine multiwavelength continuum imaging to determine the nature of this source.

\section{OBSERVATIONS}

The submillimeter continuum observations were obtained on 1996 April 28 at the CSO using SHARC, a ${ }^{3} \mathrm{He}$-cooled monolithic silicon bolometer array consisting of 24 pixels in a linear arrangement (Hunter, Benford, \& Serabyn 1996). For a source with a submillimeter spectral index of 4 , the effective frequency of the $350 \mu \mathrm{m}$ filter is $852 \mathrm{GHz}$ and the effective bandwidth is $103 \mathrm{GHz}$. Maps were obtained by scanning the telescope in azimuth, while the secondary was chopping $86^{\prime \prime}$ at $4.1 \mathrm{~Hz}$. Successive scans were made after stepping in elevation increments of 4". Flux calibration was based on Uranus. Also at $\mathrm{CSO}$, a $\mathrm{H}_{2} \mathrm{CO}_{2,1} \rightarrow 2_{1,1}(225.698 \mathrm{GHz})$ map was obtained in 1996 May via point by point mapping with a chopper throw of $5^{\prime}$ and a beam size of $31^{\prime \prime}$. A grid of 56 points was observed with a spacing of $15^{\prime \prime}$.

At the Owens Valley Radio Observatory (OVRO) Millimeter Array, G34.24+0.13 was simultaneously observed at two continuum frequencies with $1 \mathrm{GHz}$ bandwidth. The high-frequency band was tuned to $225.7 \mathrm{GHz}$ during two transits observed in 1997 February for a total of 30 baselines. The low-frequency band was tuned to $94.5 \mathrm{GHz}$ during the first transit (in lowresolution configuration) and $110.7 \mathrm{GHz}$ during the second transit (in equatorial configuration). In all cases, the phase-gain calibrator was $1741-038$ ( $18^{\circ}$ distant) and the absolute-flux calibrator was Neptune. Elevation-dependent amplitude corrections were applied to the $225 \mathrm{GHz}$ data.

Infrared observations were made with the Hale 200 inch telescope at Palomar Observatory on 1997 July 18-19. Nearinfrared images in the $Z(1.0 \mu \mathrm{m}), J(1.2 \mu \mathrm{m}), H(1.6 \mu \mathrm{m}), K_{s}(2.2$ 
$\mu \mathrm{m})$, and $L^{\prime}(3.7 \mu \mathrm{m})$ bands were obtained with the Cassegrain near-infrared camera, which contains a $256 \times 256 \mathrm{InSb}$ array detector. The pixel size was $00^{\prime \prime} 125 \mathrm{pixel}^{-1}$, and the seeing disk FWHM at $L^{\prime}$ was $0 " 6$; an $8^{\prime \prime} \times 8^{\prime \prime}$ subarray was used at $L^{\prime}$. The 10 and $20 \mu \mathrm{m}$ photometry was obtained with the singleelement bolometer photometer through a 4".6 diameter beam with the $f / 70$ secondary chopping 6 " in declination. Air-mass corrections were made to all of the infrared data. Subsequent astrometric observations were made on 1997 August 22, and further $20 \mu \mathrm{m}$ photometry was obtained on 1997 October 16.

\section{RESULTS}

The $350 \mu \mathrm{m}$ image of the G34.3+0.2 complex is shown in Figure 1 . The peak $350 \mu \mathrm{m}$ intensity coincides with the UCHII region and the primary $\mathrm{H}_{2} \mathrm{O}$ maser complex. A ridge of emission extends to the northwest and contains a local maximum coinciding with the secondary $\mathrm{H}_{2} \mathrm{O}$ maser complex (Fey et al. 1994). Of the several sources evident in the map, perhaps the most interesting is the isolated, compact source with Galactic coordinates G34.24+0.13 that lies $84^{\prime \prime}(1.5 \mathrm{pc})$ southeast of the UCHII region. In $2 \mathrm{~cm}$ maps of the region (see, e.g., Fey et al. 1994) there is no compact radio continuum emission at the position of G34.24+0.13 to an rms level of $5.7 \mathrm{mJy}$. A map of the $\mathrm{H}_{2} \mathrm{CO} 3_{2,1} \rightarrow 2_{1,1}$ transition around $\mathrm{G} 34.24+0.13$ reveals an unresolved core $\left(v_{\text {LSR }}=57 \mathrm{~km} \mathrm{~s}^{-1}\right)$ whose position is consistent with the isolated $350 \mu \mathrm{m}$ source. Emission in this transition indicates a high volume density $\left(10^{5}-10^{6} \mathrm{~cm}^{-3}\right)$ of molecular gas (Wang et al. 1995).

A more accurate position and size of the G34.24+0.13 core is obtained from the millimeter interferometric maps shown in Figure 2 (Plate L9). The source is easily detected at $225 \mathrm{GHz}$ with a deconvolved size of $2.7 \times 1$.". at the position $18^{\mathrm{h}} 50^{\mathrm{m}} 49^{\mathrm{s}} .04,+01^{\circ} 09^{\prime} 59^{\prime \prime} .5$ (equinox 1950 ) with a $0^{\prime \prime} .5$ uncertainty. It is marginally detected at a $5 \sigma$ level at $110 \mathrm{GHz}$ and undetected at $94.5 \mathrm{GHz}$. The fitted source parameters are summarized in Table 1.

The $L^{\prime}$ image was dominated by a single prominent $\left(L^{\prime} \sim\right.$ $11.7 \mathrm{mag}$ ) point source with a deconvolved size of less than 0.5 . A counterpart to the $L^{\prime}$ source was seen in two of the shorter wavelength images, growing progressively fainter: $K_{s}$ (14.8 mag), $H$ (17.1 mag), and $J(>20.1 \mathrm{mag})$. Photometry on this source yielded detections at 10 and $20 \mu \mathrm{m}$. The flux densities are listed in Table 1; much of the uncertainty results from source confusion. The coordinates of the near-infrared source were measured at the telescope by comparing the $K_{s}$ image with that of five nearby bright stars whose coordinates were obtained from the Hipparcos catalog (S. Urban 1997, private communication). This procedure yielded the position: $18^{\mathrm{h}} 50^{\mathrm{m}} 48 \mathrm{~s} 87,+01^{\circ} 10^{\prime} 01^{\prime \prime} 8$ (equinox 1950), thus placing the infrared source 3".4 away from the millimeter source, as shown in Figure 2. In the $K_{s}$ image, the millimeter source lies within a pronounced dark region devoid of the extended nebular emission seen to the north and west. We conclude that there are two distinct sources present: the millimeter source G34.24+0.13MM and the adjacent infrared source G34.24+0.13IR. Additional single beam photometry at the millimeter position yielded an upper limit of $\approx 200 \mathrm{mJy}$ at 20 $\mu \mathrm{m}$ with evidence of emission in the reference beam. We hope to obtain $20 \mu \mathrm{m}$ imaging in the near future to gain a clearer view of the region.

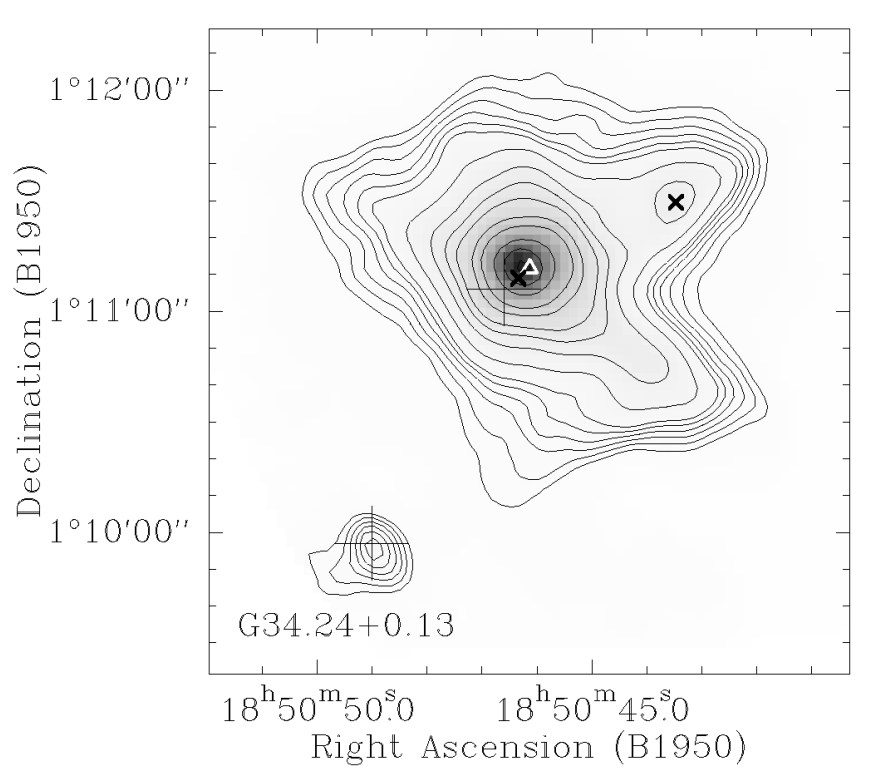

FIG. 1.-350 $\mu \mathrm{m}$ image of G34.26 +0.15 with contour levels: $14,16,18$, $20,22,24,30,40,50,60,80,100,140,200$, and $250 \mathrm{Jy} \mathrm{beam}^{-1}$. The position of the UCHII region is marked with a triangle, and the $\mathrm{H}_{2} \mathrm{O}$ masers (Fey et al. 1994) are marked by "X." The $\mathrm{CH}_{3} \mathrm{OH}$ maser positions are marked by crosses, whose size reflects the single-dish position uncertainty (Caswell et al. 1995a).

\section{DISCUSSION}

\subsection{G34.24+0.13MM: A Proto-B Star}

As shown in Figure 3, the (sub)millimeter spectrum of G34.24+0.13MM is quite steep $\left(F_{\nu} \propto \nu^{3.9 \pm 0.4}\right)$. We attribute the emission to thermal dust, which emits as a modified blackbody with $I_{\nu} \propto \nu^{2+\beta}$ in the Rayleigh-Jean regime, where $\beta$ is the power-law frequency index of the grain opacity. In this case, the long-wavelength data are consistent with $\beta=2$. Using this value, the long-wavelength spectrum can be approximated by a modified graybody with two free parameters: the dust temperature $(T)$ and optical depth at a reference wavelength $\left(\tau_{125 \mu \mathrm{m}}\right)$. Using the empirical grain opacities of Hildebrand (1983), we have fitted a modified graybody with a solid angle corresponding to the millimeter angular size (2".0). We compute an upper limit to the dust temperature by fitting for the $20 \mu \mathrm{m}$ flux limit. Using the temperature and optical depth derived from this fit, the bolometric luminosity, total mass of molecular gas, column density, and average volume density were computed (see Table 2). Because the results are sensitive to the source angular size, we computed fits for a range of sizes $\left(1^{\prime \prime}-3^{\prime \prime}\right)$ to quantify the uncertainty in the model.

The bolometric luminosity of G34.24+0.13MM (1600-6300 $L_{\odot}$ ) is similar to the range of values computed for $\mathrm{W} 3 \mathrm{OH}-\mathrm{TW}$ (Wilner et al. 1995) and corresponds to zero-age main-sequence (ZAMS) stars B3 to B0 (Panagia 1973). For the case of optically thin free-free emission, the $15 \mathrm{GHz}$ flux limit implies an excitation parameter of less than $6.2 \mathrm{pc} \mathrm{cm}^{-2}$ (Schraml \& Mezger 1969), which is inconsistent with the presence of any ZAMS star earlier than B1. The central object is either of later type or has not yet reached the ZAMS. The gas column density $\left(10^{25} \mathrm{~cm}^{-2}\right)$ and average volume density $\left(10^{8} \mathrm{H}\right.$ atom $\left.\mathrm{cm}^{-3}\right)$ are extreme even for massive star-forming regions. Also, the compact size of the core implies that it is optically thick to wavelengths as long as $\approx 200 \mu \mathrm{m}$. To summarize, G34.24+0.13MM 
TABLE 1

ObServed Continuum Emission Properties of G34.24+0.13MM and G34.24+0.13IR

\begin{tabular}{|c|c|c|c|c|c|c|}
\hline Source & $\begin{array}{l}\text { Wavelength } \\
\quad(\mu \mathrm{m})\end{array}$ & $\begin{array}{l}\text { Frequency } \\
(\mathrm{GHz})\end{array}$ & $\begin{array}{l}\text { Flux Density } \\
(\mathrm{mJy})\end{array}$ & $\begin{array}{l}\text { Beam Size } \\
(\operatorname{arcsec})\end{array}$ & $\begin{array}{l}\text { Observed FWHM } \\
\quad(\operatorname{arcsec})\end{array}$ & $\begin{array}{c}\text { Deconvolved FWHM } \\
(\operatorname{arcsec})\end{array}$ \\
\hline \multirow[t]{5}{*}{$\mathrm{G} 34.24+0.13 \mathrm{MM}$} & 3170 & 94.5 & $<13$ & $6.6 \times 3.5$ & & \\
\hline & 2710 & 110.7 & $18 \pm 9$ & $4.5 \times 3.5$ & $6.4 \times 3.3$ & $\approx 2.3$ \\
\hline & 1330 & 225.7 & $143^{\mathrm{a}} \pm 29$ & $2.7 \times 2.4$ & $3.8 \times 2.8$ & $2.7 \times 1.4\left(+127^{\circ}\right)^{\mathrm{c}}$ \\
\hline & 352 & 852 & $24000 \pm 7000$ & 12 & & \\
\hline & 20 & 15000 & $<200$ & $4.6^{\mathrm{b}}$ & & \\
\hline \multirow[t]{7}{*}{ G34.24+0.13IR $\ldots \ldots \ldots$} & 1330 & 225.7 & $<11$ & $2.7 \times 2.4$ & & \\
\hline & 20 & 15000 & $570 \pm 114$ & $4.6^{\mathrm{b}}$ & & \\
\hline & 10.6 & 28000 & $140 \pm 14$ & $4.6^{\mathrm{b}}$ & & \\
\hline & 3.7 & 81000 & $5.5 \pm 0.9$ & $2.5^{\mathrm{b}}$ & 0.73 & $<0.5$ \\
\hline & 2.2 & 140000 & $0.84 \pm 0.12$ & $2.5^{\mathrm{b}}$ & & \\
\hline & 1.6 & 190000 & $0.14 \pm 0.02$ & $2.5^{\mathrm{b}}$ & & \\
\hline & 1.2 & 250000 & $<0.015$ & $2.5^{\mathrm{b}}$ & & \\
\hline
\end{tabular}

${ }^{\text {a }}$ The flux density at $1330 \mu \mathrm{m}$ has been corrected for an $11 \%$ primary beam attenuation.

${ }^{b}$ Diameter of circular beam.

${ }^{\mathrm{c}}$ Position angle of deconvolved source (east of north).

exhibits many of the characteristics traditionally associated with protostars.

\subsection{The Nature of G34.24+0.13IR}

The bolometric luminosity of G34.24+0.13IR must be approximately $50 L_{\odot}$. We propose three possible origins for this source: (1) a reflection nebula on the outskirts of G34.24+0.13MM, (2) a background star reddened by the cold

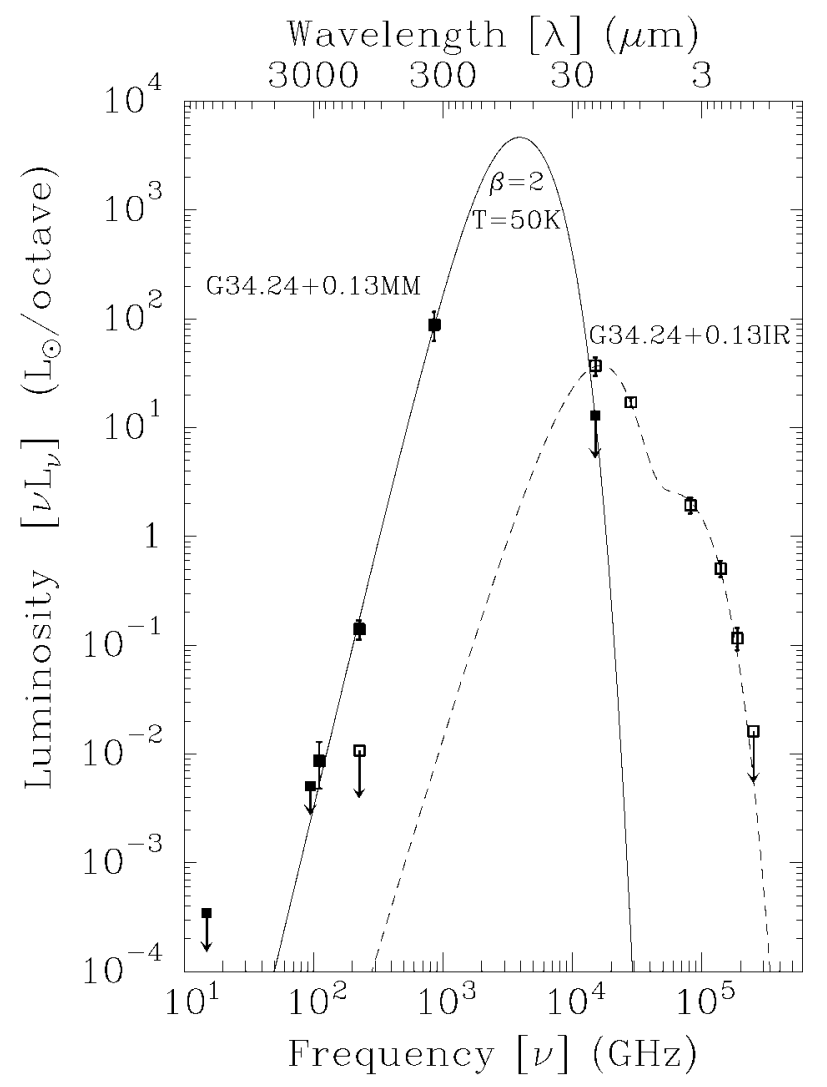

FIG. 3.-Spectral energy distribution of G34.24+0.13MM (solid squares and line) and G34.24+0.13IR (open squares and dashed line). On the vertical axis, $L_{v}$ has been taken as $4 \pi R^{2} F_{v}$ where $R=3.7 \mathrm{kpc}$. The vertical bars on the data points represent $1 \sigma$ errors on the measured flux densities. The 94.5 $\mathrm{GHz}$ and $1.2 \mu \mathrm{m}$ points are $3 \sigma$ upper limits. The $1 \sigma$ upper limit at $15 \mathrm{GHz}$ is from Fey et al. (1994). The model for G34.24+0.13IR includes a warm dust cocoon surrounding a reddened and attenuated central blackbody. dust of G34.24+0.13MM, and (3) an adjacent young star with its own circumstellar material. The first possibility may be the least likely because the source appears pointlike rather than nebulous. The other two possibilities suggest a reddened ZAMS A2 star. The expected $H-K$ color for such a star is +0.01 mag (Koornneef 1983), while the $H-K$ color of G34.24+0.13IR is $2.34 \mathrm{mag}$. The resulting excess of $2.33 \mathrm{mag}$ is consistent with an optical extinction $A_{V}=37 \mathrm{mag}$ (Rieke \& Lebofsky 1985). Using the conversion factor of $2 \times 10^{21}$ $\mathrm{cm}^{-2}\left(A_{V}\right)^{-1}$, this extinction requires a gas column density $\approx 7.4 \times 10^{22} \mathrm{~cm}^{-2}$, a factor of 2-4 below the upper limit column density of cold dust implied by the $225 \mathrm{GHz}$ nondetection. However, because the 10-20 $\mu \mathrm{m}$ color suggests much warmer dust, we favor the third case and have constructed a model with a central blackbody surrounded and attenuated by a uniform spherical dust cocoon, with the dust depicted as a modified graybody with $\beta=1$. The temperature and radii of the two components and the dust mass were fitted simultaneously with the constraint that the emergent luminosity equal that of the unattenuated central blackbody. In this model, a small mass $\left(6 \times 10^{-4} M_{\odot}\right)$ of $170 \mathrm{~K}$ dust in a cocoon of radius $55 \mathrm{AU}$ around a $1640 \mathrm{~K}$ blackbody of radius $0.85 \mathrm{AU}$ best reproduces the infrared emission.

\subsection{The $\mathrm{CH}_{3} \mathrm{OH}$ Maser Connection}

Class II $\mathrm{CH}_{3} \mathrm{OH}$ masers (such as $\mathrm{G} 34.24+0.13$ ) generally occur in the hot dense envelopes of UCHII regions (Menten et al. 1992). They mark sites of more recent star formation, in contrast to the class I variety, which tend to trace the interaction of high-velocity gas with ambient material (Mehringer \& Menten 1997). Theoretical work shows that class $\mathrm{II} \mathrm{CH}_{3} \mathrm{OH}$ maser transitions are radiatively pumped by submillimeter photons (Cragg et al. 1992). Therefore, the presence of the $\mathrm{CH}_{3} \mathrm{OH}$ maser emission is most likely associated with the bright submillimeter source G34.24+0.13MM. It is interesting to note that G34.24+0.13MM has never been identified in $\mathrm{H}_{2} \mathrm{O}$ maser surveys. As shown by statistical studies, $\mathrm{H}_{2} \mathrm{O}$ masers trace a brief evolutionary stage prior to the formation of an UCHII region (Codella et al. 1994). Thus, it is likely that G34.24+0.13MM is in a phase prior to the $\mathrm{H}_{2} \mathrm{O}$ maser phase. This status bolsters the protostellar nature of G34.24+0.13MM and supports the claim of Caswell et al. (1995b) that $\mathrm{CH}_{3} \mathrm{OH}$ masers are one of the best and most practical tracers of massive protostars. 
TABLE 2

Physical Properties of G34.24+0.13MM versus Model Angular Size

\begin{tabular}{ccccccc}
\hline \hline $\begin{array}{c}\theta_{\text {FWHM }} \\
(\operatorname{arcsec})\end{array}$ & $\begin{array}{c}T_{\text {dust }} \\
(\mathrm{K})\end{array}$ & $\tau_{125 \mu \mathrm{m}}$ & $\log \left(L_{\mathrm{bol}} / L_{\odot}\right)$ & $\log \left(M_{\mathrm{H}} / M_{\odot}\right)$ & $\log \left(N_{\mathrm{H}} / \mathrm{cm}^{-2}\right)$ & $\log \left(\bar{n}_{\mathrm{H}} / \mathrm{cm}^{-3}\right)$ \\
\hline $3.0 \ldots \ldots$ & $47^{\mathrm{a}}$ & $1.8^{\mathrm{a}}$ & 3.8 & 2.0 & 24.6 & 7.4 \\
$2.0 \ldots \ldots$ & $50^{\mathrm{a}}$ & $4.0^{\mathrm{a}}$ & 3.6 & 2.0 & 25.0 & 7.9 \\
$1.0 \ldots \ldots$ & $55^{\mathrm{a}}$ & $15^{\mathrm{a}}$ & 3.2 & 2.0 & 25.6 & 8.8 \\
\hline
\end{tabular}

${ }^{\mathrm{a}}$ Fitted parameters $(\beta=2$ in all cases).

\section{CONCLUSIONS}

Combining high-resolution 1.2-3.7, 350, 1330, and $2700 \mu \mathrm{m}$ continuum images with 10 and $20 \mu \mathrm{m}$ photometry, we have identified a deeply embedded object (G34.24+0.13MM) coinciding with the $\mathrm{CH}_{3} \mathrm{OH}$ maser G34.24+0.13. Millimeter synthesis images provide an accurate measurement of the source position and angular diameter $(2$ ". 0 , or $7600 \mathrm{AU})$. The spectral energy distribution is consistent with dust emission with a grain emissivity index of 2 and a temperature $\sim 50 \mathrm{~K}$. We estimate the total gas mass to be $100 M_{\odot}$ and the bolometric luminosity to be between 1600 and $6300 L_{\odot}$. These quantities are consistent with a deeply embedded proto-B star as the powering source of the dust emission. In view of the spectrum of $\mathrm{G} 34.24+0.13 \mathrm{MM}$, future searches for massive protostars using submillimeter dust and $\mathrm{CH}_{3} \mathrm{OH}$ maser emission are likely to be most fruitful.

We thank R. Burrows for assistance at the 200 inch, and D. Shepherd for help with the OVRO observations. The CSO and OVRO are funded by the NSF under contracts AST96-15025 and AST96-13717. We are grateful to A. Sargent, T. K. Sridharan, T. Megeath, D. Wilner, and D. Mehringer for helpful discussions. We thank S. Urban at USNO for directing us to the Hipparcos coordinates of our reference stars. T. R. H. thanks P. Ho and the SAO for supporting this research, which made use of the SIMBAD database operated at CDS, Strasbourg, France.

\section{REFERENCES}

Akeson, R. L., \& Carlstrom, J. E. 1996, ApJ, 470, 528

Benson, J. M., \& Johnston, K. J. 1984, ApJ, 277, 181

Caswell, J. L., Vaile, R. A., Ellingsen, S. P., \& Norris, R. P. 1995a, MNRAS, 274, 1126

Caswell, J. L., Vaile, R. A., Ellingsen, S. P., Whiteoak, J. B., \& Norris, R. P. 1995b, MNRAS, 272, 96

Cesaroni, R., Churchwell, E., Hofner, P., Walmsley, C. M., \& Kurtz, S. 1994, A\&A, 288, 903

Codella, C., Felli, M., Natale, V., Palagi, F., \& Palla, F. 1994, A\&A, 291, 261

Cragg, D. M., Johns, K. P., Godfrey, P. D., \& Brown, R. D. 1992, MNRAS, 259,203

Fey, A. L., Gaume, R. A., Nedoluha, G. E., \& Claussen, M. J. 1994, ApJ, 435,738

Garay, G., Reid, M. J., \& Moran, J. M. 1985, ApJ, 289, 681

Gaume, R. A., \& Mutel, R. L. 1987, ApJS, 65, 193

Heaton, B. D., Little, L. T., \& Bishop, I. S. 1989, A\&A, 213

Hildebrand, R. H. 1983, QJRAS, 24, 267
Hunter, T. R. 1997, Ph.D. thesis, Caltech

Hunter, T. R., Benford, D. J., \& Serabyn, E. 1996, PASP, 108, 1042

Koornneef, J. 1983, A\&A, 128, 84

Kuchar, T. A., \& Bania, T. M. 1994, ApJ, 436, 117

Mehringer, D. M., \& Menten, K. M. 1997, ApJ, 474, 346

Menten, K. M., Reid, M. J., Pratap, P., Moran, J. M., \& Wilson, T. L. 1992, ApJ, 401, L39

Mezger, P. G. 1994, Ap\&SS, 212, 197

Panagia, N. 1973, AJ, 78, 929

Reid, M. J., \& Ho, P. T. P. 1985, ApJ, 288, L17

Rieke, G. H., \& Lebofsky, M. J. 1985, ApJ, 288, 618

Schraml, J., \& Mezger, P. G. 1969, ApJ, 156, 269

Turner, J. L., \& Welch, W. J. 1984, ApJ, 287, L81

Wang, Y. S., Evans, N. J., Zhou, S. D., \& Clemens, D. P. 1995, ApJ, 454, 217

Wang, N., et al. 1996, Appl. Opt., 35, 6629

Wilner, D. J., Welch, W. J., \& Forster, J. R. 1995, ApJ, 449, L73

Wood, D. O. S., \& Churchwell, E. 1989, ApJS, 69, 831 


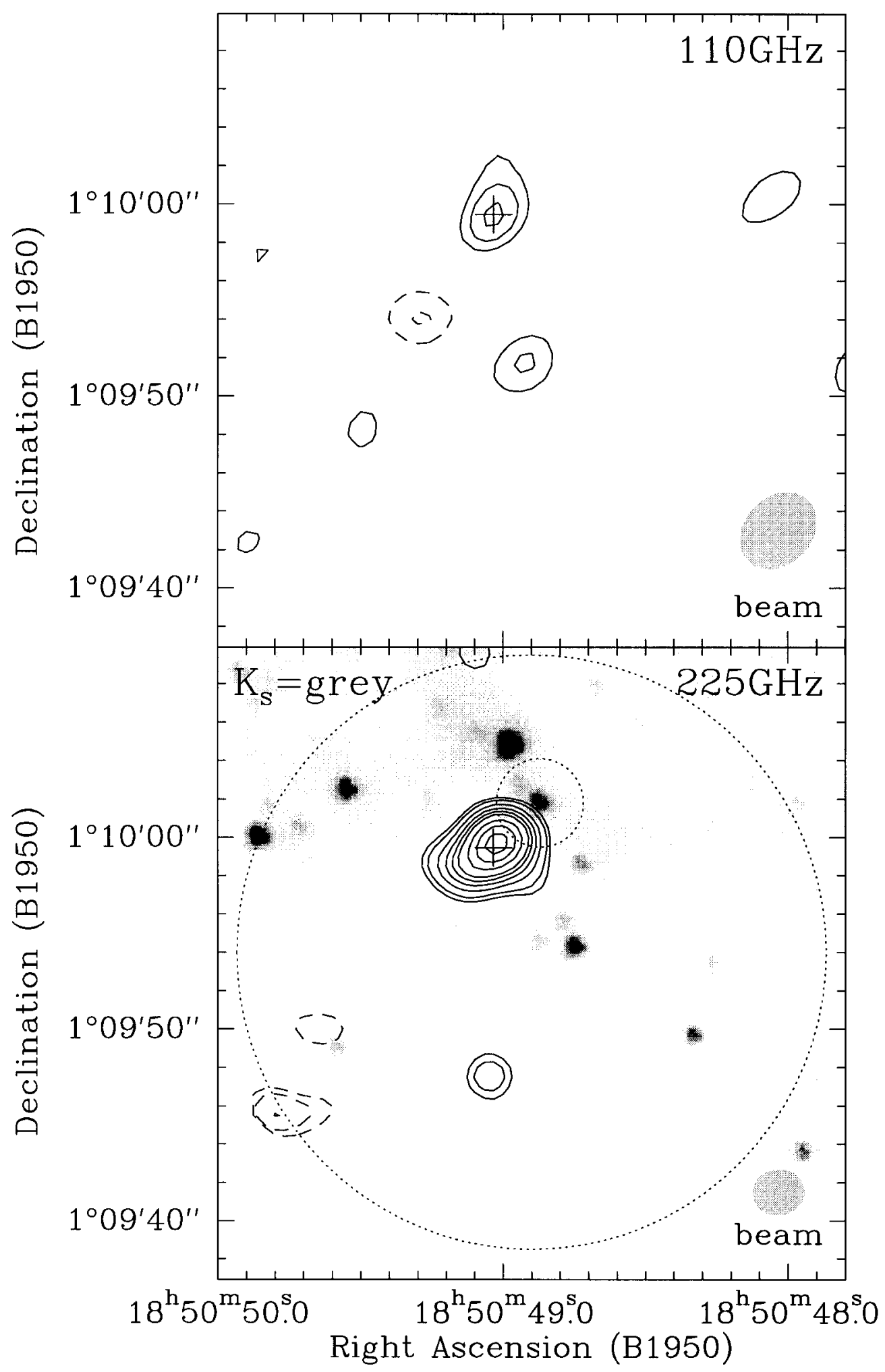

FIG. 2.-(top) OVRO $110.7 \mathrm{GHz}$ image observed with a 4".5 by 3.5 synthesized beam (natural weighting). Contour levels: $7.5,10,12.5$ mJy beam ${ }^{-1}$ (dashed contours are negative). (bottom) The Palomar $K_{s}$ image of G34.24+0.13 (gray scale) overlaid with the OVRO $225.7 \mathrm{GHz}$ continuum image (contours) observed with a 2".7 by 2".3 synthesized beam (natural weighting). The large dotted circle indicates the half-power level of the primary beam. The small dotted circle denotes the beam size and position used for the 10 and $20 \mu \mathrm{m}$ photometry of G34.24+0.13IR: $18^{\mathrm{h}} 50^{\mathrm{m}} 48 \mathrm{~s} 87,+01^{\circ} 10^{\prime} 01^{\prime \prime} 8$ (B1950). In the gray scale, stars appear dark and the small white spots result from stars in the reference image not properly subtracted. In both panels, the cross marks the fitted position of the $225 \mathrm{GHz}$ emission: $18^{\mathrm{h}} 50^{\mathrm{m}} 49^{\mathrm{s}} 04,+01^{\circ} 09^{\prime} 59^{\prime \prime} 47$ (B1950). Contour levels: 9, 12, 18, 24, 30, 36, 48, 60, $72 \mathrm{mJy}^{\text {beam }}{ }^{-1}$ (dashed contours are negative).

Hunter et al. (see 493, L98) 